\title{
Correlation Between Receptor Status and Presence of Axillary Lymph Node Metastasis in Breast Cancer in Kenya
}

\author{
Marilynn Omondi Akinyi, Kiptoon Dan, Ojuka Kinyuru Daniel, Walong Edwin
}

School of Medicine, University of Nairobi

Correspondence to: Dr. Marilynn Akinyi Omondi, Department of Surgery, University of Nairobi, PO Box 19676-00202, Nairobi; email: marokinyi@gmail.com

\begin{abstract}
Background: Breast cancer survival is linked to early detection, and timely and appropriate treatment. Survival depends on the stage and biological behaviour of the tumour. It is unclear how different molecular subtypes impact on axillary node involvement. Objective: This study sought to determine the correlation between hormonal receptor status with axillary nodal status in breast carcinoma. Methodology: A cross sectional study was carried out at Kenyatta National Hospital surgical wards and histopathology laboratory. Patients with a histological diagnosis of breast cancer, scheduled to undergo a modified radical mastectomy, were recruited. Data collected included age at diagnosis, parity, menopausal status, clinical examination findings, stage of the disease clinically and pathologically, and hormonal receptor status. Data were analyzed using SPSS version 21.0. Results: We enrolled 79 women with invasive breast carcinoma. Mean age was 48 $(\mathrm{SD}=14.5)$ years. Most (48.1\%) presented with stage III tumour.
\end{abstract}

\section{Introduction}

Breast cancer growth, spread and management are hormone dependent. Guidelines written jointly by the American Society of Clinical Oncology and the College of American Pathologists recommend the routine analysis of oestrogen and progesterone receptors (ER/PR) in all breast cancers (1). This is because the presence of these receptors determines prognosis and selection of appropriate treatment. The success of hormonal therapies such as selective oestrogen receptor modulators and aromatase inhibitors is limited to patients whose cancers express ER/PR. Low grade tumours are more likely to be ER/PR positive and tend to have a better prognosis unlike those with a negative $\mathrm{ER} / \mathrm{PR}$ status that tend to be larger, higher stage tumours with poorly differentiated morphology and an increased number of axillary lymph node metastases $(2,3)$.

Multifocal disease, high grade disease, large tumours and lymphovascular invasion have been described as predictors of axillary node metastasis, but the impact of tumour molecular subtype on axillary node involvement has not been correlated yet $(4,5)$.

Lymph node metastasis is the most important prognostic factor in patients with operable breast cancer. The number of positive lymph nodes correlates directly with local and distant recurrence. The five-year overall survival (OS) is $82.8 \%$ in
Most tumours (58.2\%) were luminal A. Correlation between molecular type and nodal involvement was not significant. Luminal B was significantly present in those above 50 years $(p=0.011)$. Conclusion: The most common molecular type of breast cancer was luminal A, but luminal B disease which was prevalent in women more than 50 years old was the only molecular subtype that had a positive correlation with axillary nodal status.

Key words: Breast cancer, Axillary lymph nodes, Molecular subtypes, Hormonal receptor status, HER-2 status

Ann Afr Surg. 2019; 16(2):51-54

DOI:http://dx.doi.org/10.4314/aas.v16i2.2

Conflicts of Interest: None

Funding: None

(C) 2019 Author. This work is licensed under the Creative Commons Attribution 4.0 International License.

lymph node negative disease but drops to $73 \%$ in patients with $1-3$ positive lymph nodes, reducing further to $45.7 \%$ in patients with $4-12$ positive nodes and to $28.4 \%$ in those with more than 13 positive lymph nodes $(6,7)$. Rates of axillary node involvement in the different molecular breast cancer subtypes based on their combined immunohistochemistry expression of hormonal receptors and human epidermal growth factor receptor 2 (HER-2) status have not been systematically described in our population, and controversies about the role of lymph node involvement as an intrinsic characteristic of breast cancer genetics remains (8).

This study sought to determine the correlation between molecular types of breast cancer and the axillary lymph node status.

\section{Patients and Methods}

A cross sectional study was conducted over 9 months at the surgical wards and histopathology laboratory of Kenyatta National Hospital. The study was carried out among consenting female patients 18 years and above with a histological diagnosis of breast cancer stages I-III scheduled to undergo modified radical mastectomy. We excluded those with previous history of lymph node dissection or those with neoadjuvant therapy and those with recurrent disease. 
Data collected included age at diagnosis, parity, menopausal status, clinical examination findings and stage of the disease, histopathologic findings including nodal status, hormonal receptor status and HER-2.

Following gross examination and selection of tissues for evaluation, the specimens were preserved for at least 6 hours in $10 \%$ neutral buffered formal saline, then processed for up to 8 hours to dehydrate the tissues and impregnate the cells with molten paraffin wax to prevent tissue degradation. The tissue section was then processed and stained with haematoxylin and eosin stain. [9] Immunohistochemistry was performed using tissue microarrays for hormonal receptor status and HER-2. This information was then used to classify invasive breast cancer into the different molecular subtypes described, which are luminal A(ER/PR + HER-2 -ve), luminal B(ER/PR +ve HER-2 + ve or -ve), HER-2 enriched (ER/PR -ve HER-2 +ve), basal like and triple negative disease (ER/PR - ve HER-2 -ve) (10).

Data were analyzed using SPSS version 21.0. Patient characteristics were summarized using age, parity and menopausal status; continuous data were presented as means and categorical variables as proportions. Prevalence of hormone receptor expression was presented as a proportion of all breast cancer patients. Axillary lymph node involvement was presented as a percentage and associated with hormone receptor status using chi-square test of associations. Odds ratios were calculated and presented as estimates for relative risk of axillary involvement in patients with hormone receptor expressions. The sample size was calculated to ensure that all statistical tests were conducted at $5 \%$ level of significance with a power of $80 \%$. This study was approved by the Institutional Review Board of the University of Nairobi.

\section{Results}

The cross sectional study comprised 79 women with invasive breast cancer who underwent a modified radical mastectomy. Their mean age was 48 years $(\mathrm{SD}=14.5)$; most $(54.4 \%)$ were in the age group 30-49 years (Fig. 1).

Study participants got their first child at the mean age of 23 years and the mean parity was 7 children. Most (64.6\%) were pre-menopausal and $35.4 \%$ were post-menopausal. All women

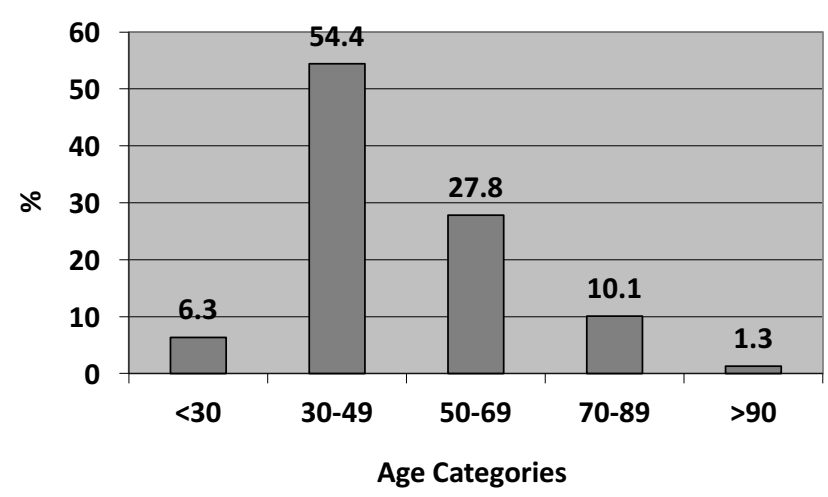

Figure 1. Age distribution had a palpable breast lump and most were T3 (55.7\%), and $25.3 \%$ were $\mathrm{T} 2$. The $\mathrm{T}$ stage of the disease in this study was based on clinical examination (10).

In the clinical nodal status, $41.8 \%$ classified as palpable and mobile, and $19 \%$ as palpable and fixed. In the pathologic staging of nodal disease, $29.1 \%$ were $\mathrm{pN} 0,19.1 \% \mathrm{pN} 1,38 \%$ $\mathrm{pN} 2$, and $13.9 \% \mathrm{pN} 3$. The P stage pathologic staging was done during histologic examination of the mastectomy specimen (10). Most (48.1\%) patients presented at stage III disease, $41.8 \%$ at stage II and only $10.1 \%$ were stage I. The histologic variants of invasive breast carcinoma were ductal carcinoma in $91.1 \%$, lobular invasive carcinoma in $6.3 \%$ and mucinous variant in $2.5 \%$. Lymph vascular invasion was found in $83.5 \%$ of all specimens submitted. Tissue microarray technology was used to carry out hormonal receptor status and HER-2 expression for all specimens submitted (Table 1).

Table 1. Hormonal receptor status

\begin{tabular}{ll}
\hline Variable & Frequency (\%) \\
\hline$E R$ & $50(63.3)$ \\
Positive & $29(36.7)$ \\
Negative & \\
$P R$ & $52(65.8)$ \\
Positive & $27(34.2)$ \\
Negative & \\
HER-2 & $12(15.2)$ \\
Positive & $67(84.8)$ \\
Negative &
\end{tabular}

Molecular classification was used to further classify patients on hormonal receptor status and HER-2 expression in breast cancer disease (Fig. 2).

Luminal A disease had the highest percentage (57.1\%) of nodal involvement of all patients with nodal disease, next was TNBC at $28.6 \%$. However, when nodal involvement was analyzed group by group, it was found to be highest in the HER-2 enriched disease group at $100 \%$, next was TNBC disease at $72.7 \%$ (Table 2).

Most patients across all age brackets had luminal A disease, next was TNBC disease (Table 2). HER-2 enriched disease was found only in the 30-49-year age bracket. Luminal B disease was prevalent in women more than 50 years old and this prevalence was statistically significant $(p=0.01)$. Most women presented with T3 disease, mostly luminal A disease (Table

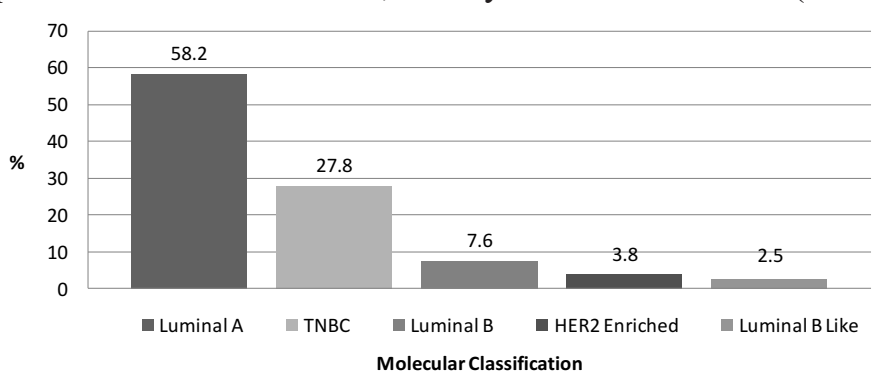

Figure 2. Molecular classification of breast cancer 
3). These results were however not statistically significant $(\mathrm{p}=0.05)$.

\section{Discussion}

Expression of ER/PR is the most reliable predictive factor for response to hormonal therapy due to its correlation with the neoplastic grade of the tumour $(2,3)$. Tumours that overexpress the HER-2 protein are associated with high-grade disease and nodal metastases $(11,12)$. Breast carcinoma will typically spread first to the axillary lymph nodes, making their assessment an integral component in the staging, prognosis and treatment of invasive breast cancer. Axillary lymph node status is an important prognostic factor for locoregional control and survival in breast cancer patients. The number of axillary lymph node metastases is an important prognostic factor when deciding on indications for adjuvant therapy. It is on this premise that the 2013 St Galen Consensus made the conclusion that luminal A breast cancer can, in most cases, be successfully treated with endocrine therapy alone. Chemotherapy is used to supplement endocrine therapy in cases of high bulk disease in this group of patients. In the different molecular subtypes of breast cancer, axillary nodal status is the most robust factor correlated to OS and Recurrence Free Survival $(7,13)$. The 5 -year OS rate for patients with lymph node metastasis is $40 \%$ less than in patients without metastasis (14-16).

The results of this study show that luminal A was the most common molecular subtype of breast cancer in Kenyan women who present at the Kenyatta National Hospital for treatment, next was TNBC. This trend ties in with several studies including that of Sayed et al. who reported the breast cancer biology pattern in African women mimics that of the western world where receptor positive disease is the most common molecular subtype $(17,18)$. Luminal A disease was spread almost evenly across all age groups. Luminal B disease was prevalent in women more than 50 years old, which was a positive correlation, and TNBC disease was found more in younger women in this study, which mirrors trends reported by Swain et al. (19). HER-2 enriched disease was expressly found in the premenopausal age group while luminal B disease was expressly found in the postmenopausal age group in this study. Slightly over $57 \%$ of the patients who had nodal disease had luminal A disease, next was TNBC disease. When nodal status was compared within the different molecular subtypes, HER-2 enriched disease followed by TNBC had the highest rates of nodal involvement at $100 \%$ and $72.7 \%$ respectively. Of patients classified as having luminal A disease, $69.6 \%$ had nodal disease. These results compare with those in studies carried out by Howland and others that show luminal A tumours have the lowest risk of lymph node metastasis while HER-2 enriched has the highest risk with the greatest number of $\mathrm{T} 1$ and T4 tumours $(13,20)$. In patients with luminal A disease, the incidence of lymph node metastasis increases as the tumour increases in size, but in TNBC disease lymph node metastasis will occur irrespective of tumour size. This trend is replicated in this study as most patients presented with $\mathrm{T} 3$ disease with a higher number of them being luminal $\mathrm{A}$, next was TNBC disease, which were prevalent across all $\mathrm{T}$ size stages. The results of the study show a trend reported by Jon Hung Lee and others that patients with luminal A disease have higher rates of nodal involvement as the T stage increased, but TNBC disease had nodal involvement regardless of the $\mathrm{T}$ size of the tumour (21). Nodal involvement rates in this study are similar to other studies that showed higher rates of lymph node positivity in hormonal receptor positive disease than in hormonal receptor negative disease $(22,23)$.

This study has demonstrated that as the T stage of the cancer

Table 2. Association between hormonal receptors, menopausal status and nodal involvement

\begin{tabular}{|c|c|c|c|c|c|c|c|}
\hline \multirow[b]{2}{*}{ Variable } & \multirow[b]{2}{*}{$n$} & \multicolumn{2}{|c|}{ Nodal involvement } & \multirow[b]{2}{*}{$P$ value } & \multicolumn{2}{|c|}{ Menopause status } & \multirow[b]{2}{*}{$P$ value } \\
\hline & & Yes (\%) & No (\%) & & Pre- & Post- & \\
\hline Luminal A & 46 & $32(57.1)$ & $14(60.9)$ & 0.760 & $30(58.8)$ & $16(57.1)$ & 0.885 \\
\hline Luminal B & 6 & $4(7.1)$ & $2(8.7)$ & 0.813 & $2(3.9)$ & $4(14.3)$ & 0.096 \\
\hline Luminal B like & 2 & $1(1.8)$ & $1(4.3)$ & 0.510 & $1(2.0)$ & $1(2.0)$ & 0.663 \\
\hline HER-2 enriched & 3 & $3(5.4)$ & $0(0.0)$ & 0.552 & $3(5.9)$ & $0(0.0)$ & 0.549 \\
\hline TNBC & 22 & $16(28.6)$ & $6(26.1)$ & 0.823 & $15(29.4)$ & $7(25.0)$ & 0.676 \\
\hline
\end{tabular}

Table 3. Association between age, size of lump and molecular subtypes of breast cancer

\begin{tabular}{|c|c|c|c|c|c|c|c|c|c|c|}
\hline \multirow[b]{2}{*}{ Variable } & \multicolumn{4}{|c|}{ Age (years) } & \multirow[b]{2}{*}{$P$ value } & \multicolumn{4}{|c|}{ Size of lump } & \multirow[b]{2}{*}{$P$ value } \\
\hline & $<30(\%)$ & 30-49 (\%) & 50-69 (\%) & $>70(\%)$ & & T1b (\%) & T1c (\%) & T2 (\%) & T3 (\%) & \\
\hline Luminal A & $1(20.0)$ & $27(62.8)$ & $12(54.5)$ & $6(66.7)$ & 0.339 & $3(100.0)$ & $5(41.7)$ & $10(50.0)$ & $28(63.6)$ & 0.241 \\
\hline Luminal B & $1(20.0)$ & $0(0.0)$ & 4 (18.2) & $1(11.1)$ & 0.011 & $0(0.0)$ & $2(16.7)$ & $0(0.0)$ & $4(9.1)$ & 0.316 \\
\hline $\begin{array}{l}\text { Luminal B } \\
\text { like }\end{array}$ & $0(0.0)$ & $1(2.3)$ & $1(4.5)$ & $0(0.0)$ & 1.000 & $0(0.0)$ & $1(8.3)$ & $1(5.0)$ & $0(0.0)$ & 0.236 \\
\hline $\begin{array}{l}\text { HER-2 } \\
\text { enriched }\end{array}$ & $0(0.0)$ & $3(7.0)$ & $0(0.0)$ & $0(0.0)$ & 0.749 & $0(0.0)$ & $0(0.0)$ & $1(5.0)$ & $2(4.5)$ & 1.000 \\
\hline TNBC & $3(60.0)$ & $12(27.9)$ & 5 (22.7) & 2 (22.2) & 0.424 & $0(0.0)$ & 4 (33.3) & $8(40.0)$ & $10(22.7)$ & 0.381 \\
\hline
\end{tabular}


increases, the chance of axillary nodal disease increases too. It has also demonstrated that luminal A disease is the most common of all molecular subtypes and has the lowest risk of nodal involvement unlike in TNBC and HER-2 enriched disease. These two subtypes seem to have nodal involvement in spite of the $\mathrm{T}$ stage of the disease. This study showed no correlation between hormonal receptor positivity or negativity and expression of HER-2 or the lack of it, and nodal status in invasive breast cancer.

\section{Conclusion}

The results show that TNBC disease regardless of T size stage will have nodal disease, and luminal A disease will have nodal disease as the T size stage increases. Almost all cases of HER2 enriched disease will invariably have nodal involvement. Luminal B disease was prevalent in women more than 50 years old, which was a positive correlation. This study has shown that by knowing the tumour size and intrinsic molecular subtype, lymph node metastasis can be predicted prior to surgery and had no direct correlation, as demonstrated by the results of the study. One can infer only by knowing the molecular subtypes of the tumour.

\section{Recommendation}

The key to successful management of breast cancer is early detection, and timely and appropriate treatment. Many different tests and methodologies need to be explored that will allow the oncologist to better and more accurately predict the biology of the cancer present, and ensure a higher survival rate. Our results did not show any significant correlation of hormonal receptor status with axillary lymph node metastasis, but more studies with larger sample sizes are needed in our setting as it is still thought that the intrinsic molecular subtype of breast cancer has an impact on the nodal status of a patient with breast cancer.

\section{References}

1. Hammond ME, Hayes DF, Wolff AC. American Society of Clinical Oncology/College of American Pathologists Guideline Recommendations for Immunohistochemical Testing of Oestrogen and Progesterone Receptors in Breast Cancer. J Oncol Pract. 2010; 6(4): 195-7.

2. Mori I, Yang Q, Kakudo K. Predictive and prognostic markers for invasive breast cancer. Pathol Int. 2002; 52:186-94.

3. Maynard PV, Davies CJ, Blamey RW, et al. Relationship between oestrogen-receptor content and histological grade in human primary breast tumours. Br J Cancer. 1978; 38:745-8.

4. Yoshihara E, Smeets A, Laenen A. Predictors of axillary lymph node metastases in early breast cancer and their applicability in clinical practice. Breast. 2013; 22(3):357-61.

5. Van Zee KJ, Kattan MW. Validating a predictive model for presence of additional disease in the non-sentinel lymph nodes of a woman with sentinel node positive breast cancer. Ann Surg Oncol. 2007; 14(8):2177-8

6. Nemoto T, Natarajan N, Bedwani R. Breast cancer in the medial half. Results of 1978 National Survey of the American
College of Surgeons. Cancer. 1983; 51:1333-8.

7. Fisher B, Bauer M, Wickerham DL. Relation of number of positive axillary nodes to the prognosis of patients with primary breast cancer: An NSABP update. Cancer. 1983; 52:1551-7.

8. Salhia B, Tapia C, Ishak EA. Molecular subtype analysis determines the association of advanced breast cancer in Egypt with favourable biology. BMC Women's Health. 2011; 11:44.

9. Slaoui M, Fiette L. Histopathology procedures: From tissue sampling to histopathological evaluation. Methods Mol Biol. 2011; 691:69-82.

10. Breast cancer staging system: AJCC Cancer Staging Manual, 8th edition. 2017

11. Pietras RJ, Arboleda J, Reese DM, et al. HER-2 tyrosine kinase pathway targets estrogen receptor and promotes hormoneindependent growth in human breast cancer cells. Oncogene. $1995 ; 10: 2435-46$.

12. Johnston S, Johnson L, Dowsett M, et al. HER-2 status in primary breast cancer patients treated in the UK TACT trial: Relationship with tumour size, grade, nodal involvement and ER status. Breast Cancer Res Treat. 2003; 82

13. Howland NK, Driver TD, Sedrak MP, et al. Lymph node involvement in immunohistochemistry-based molecular classifications of breast cancer. J Surg Res. 2013; 185:697-703.

14. Dings PJ, Elferink MA, Strobbe L, et al. The prognostic value of lymph node ratio in node-positive breast cancer: A Dutch nationwide population-based study. Ann Surg Oncol. 2013; 20:2607-14.

15. Wu SG, He ZY, Li Q, et al. Prognostic value of metastatic axillary lymph node ratio for Chinese breast cancer patients. PloS One. 2013; 8:e61410.

16. Rosen PR, Groshen S, Saigo PE, et al. A long-term follow-up study of survival in stage I (T1N0M0) and stage II (T1N1M0) breast carcinoma. J Clin Oncol. 1989; 7:355-66.

17. Sayed S, Moloo Z, Wasike R, et al. Is breast cancer from SubSaharan Africa truly receptor poor? Prevalence of ER/PR/ HER-2 in breast cancer from Kenya. Breast. 2014; 23(5):591-6.

18. Adebamowo CA, Famooto A, Ogundian TO, et al. Immunohistochemical and molecular subtypes of breast cancer in Nigeria. Breast Cancer Res Treat. 2008; 110(1):183-8.

20. Swain S. Triple-negative breast cancer: Metastatic risk and role of platinum agents. Proceedings of the ASCO Clinical Science Symposium June 3, 2008.

21. Van Calster B, Vanden Bempt I, Drijkoningen M, et al. Axillary lymph node status of operable breast cancers by combined steroid receptor and HER-2 status: Triple positive tumours are more likely lymph node positive. Breast Cancer Res Treat. 2009; 113(1):181-7.

22. Jong HL, Sung HK, Young JS, et al. Predictors of axillary lymph node metastases (ALNM) in a Korean population with T1-2 breast carcinoma: Triple negative breast cancer has a high incidence of ALNM irrespective of the tumour size. Cancer Res Treat. 2010; 42(1):30-6.

23. Lee MK, Varzi LA, Chung DU, et al. The effect of young age in hormone receptor positive breast cancer. BioMed Res Int. 2015.

24. Viale G, Zurrida S, Maiorano E, et al. Predicting the status of axillary sentinel lymph nodes in 4351 patients with invasive breast carcinoma treated in a single institution. Cancer. 2005; 103:492500. 